\title{
Probiotics: Novel Preventive and Therapeutic of Choice in an Antibiotic Resistance Era
}

\author{
Shrihari TG*, Vasudevan Vijeev, Naina S, Devraj D and Ravikumar Yella \\ Department of Oral Medicine, Krishnadevaraya College of Dental Sciences \& Hospital, Indiac \\ *Corresponding author: Shrihari TG, Assistant Professor, Department of Oral Medicine, Krishnadevaraya College of Dental Sciences \& Hospital, \\ Bangalore, India, Tel: 91-9844386188; Email: drshrihariomr@gmail.com
}

Submission: 眥 April 20, 2018; Published: 眥 May 04, 2018

\begin{abstract}
Growing concerns of probiotics in recent times is due to their wide spectrum of activities with harmless effects compared to antibiotics. Live microorganisms ingested in certain quantity is beneficial for the health of human beings for their vast and versatile mechanism of actions from preventive and therapeutic action for reducing inflammation, infection and in cancer prevention by their immune stimulatory activity, anti-cancer activity and anti-microbial activity . Probiotics role in dentistry is limited and used in the treatment of various diseases is put forth in this article. Can probiotics replace antibiotics in near future in this antibiotic resistance era.

Keywords: Probiotics; Lactobacillus; Bifidobacterium B; Streptococcus salivarius subspecies; Dental caries; Periodontal diseases; IgG; IgM; NK cells; Immune stimulation; Anticancer activity

Abbreviations: WHO: World Health Organization; FAO: Food and Agriculture Organization; CFU: Colony Forming Units; TGF- $\beta$ : Transforming Growth Factor Beta; IL: Interleukins; TNF $\alpha$ : Tumor Necrosis Factor Alpha; IFN $\gamma$ : Interferon Gamma; IgG, IgM, IgA: Immunoglobulin G, Immunoglobulin M, Immunoglobulin A; NK cells: Natural Killer Cells; VSC: Volatile Sulphur Compounds; CH3SH: Methyl Mercaptan; H2S: Hydrogen Sulphide; GRAS: Generally Recognized As Safe; SM: Streptococcus Mutans; PG: Porphyromonas Gingivalis; PI: Prevotella Intermedia; LGG: Lactobacillus rhamnosus GG; CA: Candida Albicans; AA: Aggregatibactor Actinomycetemcomitans; DNA: Deoxyribonucleic Acid; TH1: T Helper lymphocytic type 1; TH2: T Helper lymphocytic type 2
\end{abstract}

\section{Introduction}

All the bacteria are not detrimental to the human body. Some microbes have beneficial health effects on the host. These live microbes are termed as probiotics [1]. Probiotics are the dietary supplements containing potentially beneficial bacteria or yeasts. These products help to activate health promoting flora and also suppress the pathologic colonization and disease spread [2].

The term 'probiotic' was extracted from the Greek word meaning "for life" [3]. The pioneers in using the term in 1965 were Lilley and Stillwell for describing substances secreted by one organism which stimulates the growth of another. Ukrainian Nobel prize laureate, Sir Elie Metchnikoff who was working, suggested that the prolonged life of Bulgarian peasants occurred due to their consumption of fermented milk products due to lactic acid bacteria can render a great service to intestinal putrefaction and might ameliorate old age [4].

An expert panel commissioned by the World Health Organization (WHO) and Food and Agriculture Organization (FAO) defined probiotics as "live micro-organisms", which when administered in adequate amounts confer a health benefit on the host [5]. An International Life Science Institute, Europe consensus document

proposed an easy and widely accepted definition of probiotics as "Viable microbial food supplements that beneficially influence the health of human" [6]. The guidelines proposed by FAO/WHO for evaluation of probiotics advocated that every potential probiotic strains have to be correctly identified using both phenotypic and genotypic methods and followed by different tests to investigate its survival ability and functional properties [7].

Antibiotics not only destroy harmful bacteria's but also beneficial commensals of our body including alteration in normal oral microflora. Inadvertent use of antibiotics leads to many local (Oral cavity) such as acute atrophic candidiasis and systemic adverse effects such as antibiotic associated diarrhoea and antibiotic resistance, which is a major cause of death worldwide. Antibiotic resistance kills many lives globally every year; we are running short of antibiotics to microorganisms because of resistance. Antibiotic resistance is a major cause of concern at present situation in this post-antibiotic era and that to find an alternative to antibiotics is in near future. Hence, Probiotics came in to picture in this scenario, which is relatively safe by enhancing the beneficial microflora of oral cavity. This article highlights about probiotics and their role in various diseases of oral cavity, future implications. 


\section{Probiotic species [Table 1];[8]}

Table 1: Proven probiotic microorganisms [8].

\begin{tabular}{|c|c|c|}
\hline Lactobacillus Species & Biffidobacterium Species & Non Lactic Acid Bacteria \\
\hline L.rhamnosus GG (LGG) & B.bifidum & Pediococcus \\
\hline L.acidophilus LA-1 & B.longum & W.Cibaria \\
\hline L.rhamnosus GR-1 & B.thermophilum & S.cremoris \\
\hline L.casei DN114001 & B.lactis $\mathrm{Bb} 12$ & E.faecium \\
\hline L.plantarum 299V & B.longum BB536 & Escherichia Coli strain nissle \\
\hline L.acidophilus LB & B.lactis HN019 & S.salivarius subsp thermophilus \\
\hline L.casei shirota & B.breve strain Yakult & Leuconostoc \\
\hline L.acidophilus NCFM & B.infantis 35624 & Saccharomyces boulardiilyo \\
\hline L.fermentum VR1003 & B.animalis DN117-001 & S.intermedius \\
\hline L.rhamnosus HN001 & B.adolescentis & S.diaocetylactis \\
\hline L.reuteri RC-14 & B.thermophilum & Lactococcus lactis \\
\hline L.paracasei F 19 & B.infantis & Propionibacterium \\
\hline L.salivarius UCC118 & Bifidobacterium DN-17301 & Subsp.cremoris \\
\hline L.cellobiosus & B.breve & \\
\hline L.johnsonii LJ-1 & B.lactis & \\
\hline \multicolumn{3}{|l|}{ L.brevis } \\
\hline \multicolumn{3}{|l|}{ L.gasseri } \\
\hline \multicolumn{3}{|l|}{ L.reuteri SD 2112} \\
\hline \multicolumn{3}{|l|}{ L.lactus } \\
\hline L.sporogenes & & \\
\hline
\end{tabular}

Lactobacillus species: There are about 100 species of Lactobacilli that have been identified. They are ubiquitous gram positive, catalase negative, spore forming, fermentative, chemoorganotrophic and microaerophilic, appear as coccobacilli or rods. They are found in environment with rich carbohydrate, helpful in the protein and carbohydrate digestive enzymes, synthesizing vitamin $\mathrm{K}$, vitamin B complex and bile salts break down. Fermented foods like yogurt and dietary supplements contain this bacteria $[9,10]$.

Bifidobacterium species: There are about 30 species of bifidobacteria, predominantly present in the large intestine, rod shaped, anerobic bacteria. They generate lactic ions from lactic acid by metabolism of lactose and also help in synthesis of vitamins. They produce beneficial short chain fatty acids by indigestible carbohydrates fermentation $[11,12]$.

Streptococcus thermophilus and lactobacillus bulgaricus: These species are used in yoghurt production. They are found in intestinal tract; produce large quantities of lactase enzyme for metabolism of lactose, extremely useful for prevention and improvement of lactose intolerance and have antimicrobial activity [13].

Saccharomyces boulardi: This is the lactic acid producing non-colonizing only yeast probiotic. They secrete proteases that break down bacterial enterotoxins and their binding to intestinal receptors is inhibited. They also synthesize vitamin production and reduce serum cholesterol level. They are useful for preventing and treating antibiotic associated and traveller's diarrhoea and prevent the C. difficile recurrence, treatment of acne, reduce the $H$. pylori treatment associated side effects [14].

\section{Characteristics of microorganisms which act as probiotics}

a) Probiotic must contain minimum $30 \times 10^{9} \mathrm{CFU} /$ gram, should be acid resistant, bile resistant and confer positive health benefit on the host.

b) It should have faster multiplication and high survival rate.

c) It should posses the activity of faster and firm adherence.

d) It should reduce or exclude adherence of pathogen.

e) Produce antibacterial substances such as lactic acid, peroxide, and bacteriocins.

f) Safe, non carcinogenic, non pathogenic, non invasive.

g) Balanced flora is formed by co-aggregation.

h) In order to deliver alive into the intestine, the probiotics should sustain enough to withstand the duration of manufacturing, processing, and packing $[15,16]$. 


\section{Mechanism of action of probiotics [Figure 1]}

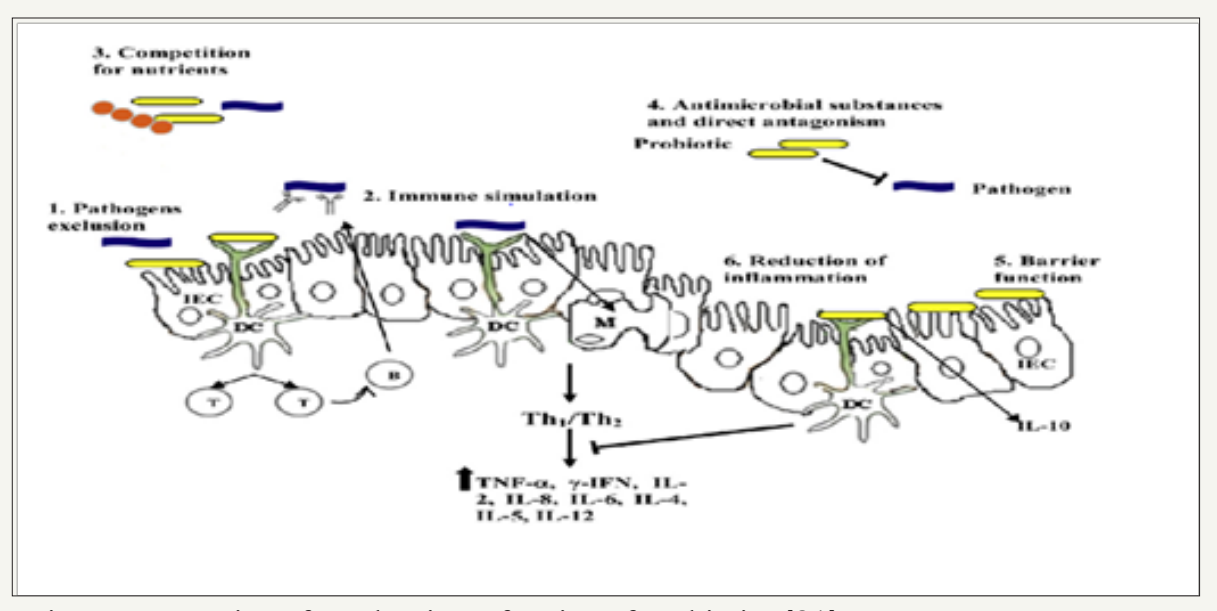

Figure 1: Diagrammatic representation of mechanism of action of probiotics [21].

a) Compete of probiotics for growth factors and nutrients.

b) Antimicrobial compound production like bacteriocins hydrogen peroxide, dipicolinic acid and organic acid which inhibits the pathogenic growth of bacteria.

c) Compete for the adhesion sites.

d) By biofilm formation, modifies microbial population.

e) Alter immune response of the host by an increase in TGF- $\beta$, IL-10, decrease in TNF- $\alpha$ and an increase in the production of $\operatorname{IgA}$.

f) Decreases inflammation associated molecules by production of short chain fatty acids (Butyrate and lactate).

g) Prevents apoptosis induced by cytokines.

h) Balances the oral and gut microbial flora, thus making its colonization resistant to pathogenic bacteria.

i) Antioxidant activity

j) Antigenotoxic activity

k) Protection of intestinal barrier integrity and mucin production

l) Increases the absorption of calcium and other minerals.

m) Modifies the structure and function of intestinal epithelium and improves the bacterial function.

n) Inhibits the pathogenic enteric bacterial growth by decreasing luminal $\mathrm{pH}$.

o) Lowers the mutagenic and toxigenic reaction by its metabolic product.

p) Increases the enterocytes turnover by butyric acid production [1,17-21].

\section{Methods of probiotic administration}

Ice creams, tablets, cheese, yoghurt, mouth rinse, capsule, liquid and lozenges [1].

\section{Probiotics in oral health}

Dental caries: Dental caries is a microbial disease of the enamel, dentin and pulp of the tooth which is caused by Streptococcus bacteria. Probiotics reduce the level of Streptococcus mutans by competing with cariogenic microbes for adhesion sites, nutrients and growth factors leading to reduced level of $S$. mutans in oral cavity [22]. Lactobacillus and Streptococcus thermophilus are the only species that have the capacity to integrate into a biofilm present on a hydroxyapatite surface and interfere with cariogenic species development. L. rhamnosus GG, L. reuteri and Streptococcus sobrinus probiotic species inhibit cariogenic streptococcal pathogens colonization reducing the incidence of tooth decay in children [14]. Many studies have shown that probiotic species in yoghurt consumption particularly L. reuteri for 2 weeks reduce the $S$. mutans count in saliva up to $80 \%$ [23]. In other comparative study, patients taking probiotics in the form of tablet or liquid showed significant reduction of S.mutans level [24]. These indicate the potential benefits of probiotics in prevention of dental caries.

Periodontal diseases: Microbial disease involving periodontium includes gingival and periodontal tissue. This is caused by P. gingivalis, T. denticola, T. forsythia and A. actinomycetem comitans, manifests as gingivitis and destruction of all supporting tissues of teeth and alveolar bone [25]. The virulent bacterial organisms evade the immune attack by colonizing in subgingival tissue and cause tissue damage [26]. Many studies have shown that using particular probiotic species in gingivitis and periodontal disease stages, pregnancy gingivitis, results in normalization of microflora, reduced bleeding in gingivitis, reduction in periodontal pathogens like Actinomyces species, Bacteroides species, Staphylococcus intermedius and Candida albicans after periodontal 
dressing with L. reuteri [27]. L. acidophilus probiotic strains periodontal micro organisms are bacterias present in supra and subgingival biofilm. Periodontal status of patients with periodontal disease is improved after using probiotics in the form of chewing gums or lozenges [22].

Oral candidiasis: It is the most common mycotic infection affecting the oral cavity. Candida species are normal commensal of the oral cavity in about $50 \%$ of healthy individuals. But in the elderly and in the immune compromised state of individuals where local or systemic immunity state is breached, it can cause a clinically apparent lesion. Many studies regarding the use of probiotics in the management of candidiasis, one among this is a randomized placebo-controlled trial use of $L$. rhamnosus $G G$ containing probiotic cheese showed prevalence in Candida albicans species [28]. The adverse effect of hyposalivation and dry mouth was noted in patients on probiotics. In another study by Koll, Lactobacillus strains of probiotic species of oral cavity inhibit the growth of periodontal pathogens such as A. Actinomycetem comitans, P. ginigivalis, P. intermedia, and S. mutans. But it has no effect on $C$. albicans growth. There is no explanation for no effect of probiotics species on Candida albicans, further investigation are needed to confirm the fact [24].

Immune enhancement: Many studies revealed specific strains of probiotics consumed in certain quantity modulate adaptive and natural immune responses. In the host, colonic microflora affects both systemic and mucosal immunity through absorption of soluble antigen or Lactobacilli translocation into the blood stream through the gut. Human intestinal epithelial cell adherence by Lactobacilli, activate blood leucocytes, macrophages, intestinal epithelial cells, $\mathrm{B}$ and $\mathrm{T}$ lymphocytes, immune system accessory cells are probiotic target cells [29].

\section{Innate immune response and probiotics}

Many studies have shown that probiotics enhance the phagocytic activity of blood leucocytes with specific strains of Lactobacillus johymsonii Lal/ Bifidobacterium lactis Bb12 for three weeks [30]. Termination of consumption of probiotics showed an increase in the sustained phagocytic activity of granulocyte for several weeks, increase in complement receptors and receptors for phagocytes in phagocytic cells on consumption of specific strains of probiotics such as lactic acid bacteria. Improvement in immune status depends on the administration of a specific quantity of $10^{9}$ CFU/day probiotics in a dose dependent manner [31]. Increase activities of NK cells was observed on administration of probiotic species L.rhamnosus or B lactis for 3 to 6 weeks along with increased phagocytic activity of blood leucocytes (neutrophils and monocytes) [32-34].

\section{Humoral immune response of probiotics}

Systemic or oral immunization potentiated humoral immune response to natural infection is increased by intake and specific strains of lactic acid bacteria such as Lactobacillus GG which induced secretion of immunoglobulins IgA, IgG, IgM after probiotic administration to infants compared to placebo given. Secreting cell of IgA specific antibody to rotavirus and increase IgA stimulation by specific probiotic strain such as Lactobacillus GG compare to L.casei subspecies rhamnosus or an S. thermophilus and L. delbruckii subspecies bulgaricus combination [35]. Mucosal or systemic vaccination immunogenicity is enhanced by probiotics. Increase in serum IgA antibody about four fold after Salmonella typhi immunization with those who consumed B. bifidum and L. acidophilus La 1containing yoghurt compared to control group where only 2.5 fold increases was found reported by Link-amster and colleagues [36]. Specialised recognition pattern expressed on immunocompetent cells (macrophages, monocytes, dendritic cells) known as pattern recognition receptors belong to toll- like receptors, present in lamina propria or peyer's patch or other sites identifies the molecular patterns of probiotics (Example: peptidoglycan, bacterial DNA, lipotechoic acid) and activates the release of various cytokines involved in developing active immune response. Antigen transportation across the gut and enhanced antigen presenting cell function activates humoral immune response; exact mechanism still needs to be understood $[34,37]$.

\section{Anticarcinogenic properties of probiotics}

Probiotics inhibit the conversion of procarcinogens into carcinogens stated by many studies, which further reduce the colon cancer risk, mutagenic compounds inactivation, antimutagenic compounds production, procarcinogenic bacterial growth inhibition or suppression, intestinal mutagen absorption reduction and immune enhancement [38]. Beta glucuronidase and nitroreductase enzymes can convert procarcinogen into carcinogens produced by Escherichia coli and Clostridium perfringes enteropathogens. Bifidobacteriumn species acts as an antibacterial or bactericidal to enteropathogens by producing lactic acid, decreasing intestinal $\mathrm{pH}$ and bacterial enzyme modification by a favourable environment. Purified cell wall of bifidobacterial has antitumor activity and induces phagocytic activity to destroy tumor cells [39].

RAS oncogene activation is the earliest and most frequent genetic alteration seen in colon cancer, cell proliferation, nuclear aplasia, histologic grade and degree of undifferentiating correlated with increase ras-21. B. longum suppressed colonic mucosal P-21 expression and tumours compared with control diet [40]. B. longum also acts as immunomodulator and modifier of biological response in tumor suppression. Probiotics producing end products acts as apoptotic inducer [41].

Halitosis: Affecting large group of the population with local and systemic factors. The main local etiological factors are chronic periodontitis, tongue coating, deep dentinal caries, poor oral hygiene and faulty restorations. Bad breath or halitosis is due to the gram positive and gram negative anaerobic organisms present on the dorsal surface of the tongue and in periodontal pockets produces volatile sulphur compounds. Replacement of pathogenic bacterias with probiotic bacterial strains may act as a potential adjuvant in prevention and treatment of halitosis [42]. A definitive reduction in the volatile sulphur compounds (VSC) producing 
bacteria F. nucleatum by ingestion of probiotic strain W. cibaria both in vivo and in vitro.

Marked reduction in the levels of $\mathrm{CH} 3 \mathrm{SH}$ and $\mathrm{H} 2 \mathrm{~S}$ was seen after gargling with probiotic W. cibaria containing rinse [27]. The possible mechanism is due to the production of hydrogen peroxide by W. cibaria, inhibits the VSC producing F. nucleatum proliferation, S. salivarius oral probiotic species inhibits the VSC producing pathogenic bacterias by competing with species increasing production of VSC for colonization sites. Probiotic strains such as E. coli Nisle 1917, S. salivarius K12, W. confusa isolates and mixture of lactic acid producing bacterias are used for mouth and gut associated halitosis [43].

AIDS: Studies done by Lin et al have shown Lactobacilli strains slow down the progression of AIDS due to binding of Lactobacili strain to the type of sugar mannose by mannose binding protein particles, found on envelope of HIV which enable the bacteria to stick and colonize to the mucosal lining of mouth and digestive tract and prevents the spread of disease by binding and colonization of HIV by forming clump of immune cells [44].

Oral cancer: Oral cancer is a seventh most common cancer in the world due to tobacco chewing habits, alcohol and smoking. Probiotics play an important role in prevention and inhibiting progression of cancer by its anti-inflammatory, antioxidant, immune-stimulatory and anti-genotoxic activity [44-46].

\section{Potential applications in dentistry}

Its role in other areas of dentistry such as in treatment of oral ulcers, premalignant lesions and conditions, immunological mucosal diseases, wound healing after extraction, bacterial infections of the oral cavity; oral cancer prevention and other oral mucosal lesions need to be studied through randomized controlled trials and long term studies.

\section{Safety and dosage}

According to the United States Food and Drug Administration, criteria for probiotic strains to be generally considered as safe (GRAS) are as follows:

\section{It should not cause systemic infections.}

2. It should not cause excessive immune stimulation and deleterious metabolic activities.

3. It should not induce pathogenic antibiotic resistance [45].

The probiotics should rather be able to maintain genetic stability in oral microflora [46] Bifidobacteria and Lactobacilli are considered to be safe, rarely induce infections, so they are in the category of Generally Recognized as safe (GRAS). But Enterococcus, Bacillus, and Streptococcus groups cause infections in humans. Analysis of potential probiotic strains should be done before their commercial applications. There is no recommended standard dosage for the probiotic administration. Studies have shown the dosages safe for probiotic strains. Dosages for Lactobacillus, ranged from 100 million to 1.8 trillion CFU/day. Dosages for saccharomyces, ranged between $250 \mathrm{mg}$ and $500 \mathrm{mg} /$ day. Depending on the age, variations in dosages are seen. Dosages for children are half of that of the adult dose. For infants, it is one fourth of the adult dose [47].

A 2008 review of probiotics noted that probiotic strain Lactobacillus rhamnosus GG application in a variety of conditions are found to be safe. A recent review reported that long term, cumulative effect of probiotics species Lactobacillus and Bifidobacterium use in children are not known and also noted that use of probiotics in critically ill patient should not be done [48]. Data is limited regarding long term safety and risk of serious side effects which can be greater in patients with underlying health conditions.

\section{Risks associated with clinical use of probiotics}

Sepsis in children; endocarditis, pneumonia and liver abscess in adults caused by Lactobacillus species are rare but in absence of probiotic supplementation, it was well recognized. Rautio et al. [49] reported liver abscess and pneumonia in a 75 year old woman using Lactobacillus GG after 4 months of daily consumption of Lactobacillus GG [49]. Mackay et al reported the case who suffered from L.rhamnosus induced endocarditis in a patient taking daily probiotic capsules after dental extraction in a 67 year old man [50]. Underlying immunocompromised chronic disease or debilitating patients on probiotics have suffered from bacteremia or fungemia. No cases of sepsis after the use of probiotics in healthy patients have been reported. The majority of cases with sepsis after probiotic consumption have resolved with appropriate antimicrobial therapy, but in some cases, patients have developed septic shock.

\section{Conclusion and Future perspectives}

Different probiotic strains will have a different mechanism of action. The optimal amounts of live probiotic bacteria to be administered have to be fixed. Isolation of a particular strain of species and their interaction with other varied different microbial species is necessary for their clinical application. The dosage of administration and time span of strain resides in that environment after administration. Interaction of probiotic strain with other diet and their mechanism of action should be elucidated for future therapeutic applications in an antibiotic resistance era.

\section{References}

1. Rastogi P, Saini H, Dixit J, Singhal R (2011) Probiotics and oral health. Natl J Maxillofac Surg 2(1): 6-9.

2. Nagaraj T, Ravi B, Sankara SN, Madhu K (2012) Probiotics and Oral Health. J Indian Aca Oral Med Radiol 24(2): 146-148.

3. Reid G, Jass J, Sebulsky MT, McCormick JK (2003) Potential use of probiotics in clinical practice. Clin Microbiol Rev 16(4): 658-72.

4. Metchnikoff E (1907) The prolongation of life. In: Heinemann W (Ed.), Optimistic studies. GP Putnam \& Sons, London, UK, pp. 1-100.

5. FAO/WHO (2001) Report of a Joint FAO/WHO Expert consultation on evaluation of health and nutritional properties of probiotics in food including powder milk with live lactic acid bacteria.

6. Salminen S, Bouley C, Boutron-Ruault MC (1998) Gastrointestinal physiology and function-targets for functional food development. Br J 


\section{Nutr 80(1): S147-S171}

7. (2002) Joint FAO/WHO working group report on drafting guidelines for the evaluation of probiotics in food London, Ontario, Canada.

8. Sameer SF, Priya M, Surabhi B, Amisha S (2016) Role of probiotics in human health and disease: An update. Int J Curr Microbiol App Sci 5(3): 328-344.

9. Patil MB, Reddy N (2006) Bacteriotherapy and probiotics in dentistry KSDJ 2: 98-102.

10. Meurman JH (2005) Probiotics: do they have a role in oral medicine and dentistry. Eur J Oral Sci 113(3): 188-196.

11. Galdeano CM, De Moreno De LA, Vinderola G, Bonet ME, Perdigón G (2007) Proposed model: mechanisms of immunomodulation induced by probiotic bacteria. Clin Vaccine Immunol 14(5): 485-492.

12. Nicole MR, Martjin BK (2000) Effects of probiotic bacteria on diarrhea lipid metabolism and carcinogenesis: a review of papers published between 1988 and 1998. Am J Clin Nutr 71(2): 405-411.

13. Soccol CR, De Souza Vandenberghe LP, Spier MR, Medeiros ABP Yamaguishi CT, et al. (2010) The potential of probiotics: A review. Food Technol Biotechnol 48(4): 413-434.

14. Kedar S, Shashikant MC, Priya T, Sultana N, Nallan CSK (2010) ProbioticsDo they have a role in medicine and dentistry. J Assoc Physicians of India 58: 488-492.

15. Azizpour K, Bahrambeygi S, Azizpour A (2009) History and basic of probiotics. Res J boil sci 4: 409-426.

16. Behnsen J, Deriu E, Sassone-Corsi M, Raffatellu M (2013) Probiotics: properties, examples, and specific applications. Cold Spring Harb Perspect Med 3(3): a010074.

17. Narwal A, Shashi Bala (2011) Probiotics in dentistry - A Review. J Nutr Food Sci 1: 114

18. Tiwari G, Tiwari R, Pandey S, Pandey P (2012) Promising future of probiotics for human health: Current scenario. Chron Young Sci 3: 17-28.

19. Calafiore A, Gionchetti P, Calabrese C, Tambasco R, Fornarini GS, et al. (2012) Probiotics, prebiotics and antibiotics in the treatment of inflammatory bowel disease. Journal of Gastroenterology and Hepatology Research 1(6): 97-106.

20. Kumar A, Vandana (2013) Probiotics: Nature's medicine. Int J Nutr Pharmacol Neurol Dis 3: 219-28.

21. Saad N, Delattre C, Urdaci M, Schmitter JM, Bressollie P (2013) An overview of the last advances in probiotic and prebiotic field. LWT - Food Science and Technology 50(1): 1-16.

22. Singh VP, Sharma J, Babu S, Rizwanulla, Singla A (2013) Role of probiotics in health and diseases: A review. J Pak Med Assoc 63(2): 253-257.

23. Nikawa H, Makihira S, Fukushima H, Nishimura H, Ozaki K, et al. (2013) Lactobacillus reuteri in bovine milk fermented decreases the oral carriage of mutans streptococci. Int J Food Microbiol 95(2): 219-223.

24. Flichy-Fernández AJ, Alegre-Domingo T, Peñarrocha-Oltra D, PeñarrochaDiago M (2010) Probiotic treatment in the oral cavity: An update. Med Oral Patol Oral Cir Bucal 15(5): 677-680.

25. Houle MA, Grenier D (2003) Maladies parodontales: connaissances actuelles current concepts in periodontal diseases. Méd Mal Infect 33 331-40.

26. Rinkee M, Bianca N, Neha S (2011) The potential role of probiotics in periodontal health. RSBO Revista Sul-Brasileira de Odontologia 9: 85-88.

27. Jaddu Jyothirmai R, Naganandini S, Shankar A (2010) Probiotics in dentistry: review of the current status. Revista de clinica e pesquisaodontologica 6: 261-267.
28. Hatakka K, Ahola AJ, Yli-Knuuttila H, Richardson M, Poussa T, et al. (2007) Probiotics reduce the prevalence of oral Candida in the elderly a randomized controlled trial. J Dent Res 86(2): 125-130.

29. Berg RD (1983) Translocation of indigenous bacteria from the intestinal tract. In: Hentges DJ (Ed.), Human intestinal Microflora in health and diseases. Academic Press, New York, USA, pp. 333-352.

30. Schiffrin EJ, Rochar F, Link-Amster H (1995) Immunomodulation of human blood cells following the ingestion of lactic acid bacteria. J Dairy Sci 78(3): 491-497.

31. He F, Tuomola E, Arvilommi H (2000) Modulation of humoral immune response through probiotic intake. FEMS Immunol Med Microbiol 29(1): 47-52.

32. Gill HS, Ruther FKJ, Gopal P (2001) Enhancement of immunity in the elderly by dietary supplementation with the probiotic Bifidobacterium lactis HN019. Am J Clin Nutr 74(6): 833-839.

33. Gill HS, Cross ML, Rutherfurd KJ (2001) Dietary probiotic supplementation to enhance cellular immunity in the elderly. $\mathrm{Br}$ J Biomed Sci 58(2): 94-96

34. Gill HS (1998) Stimulation of the immune system by lactic cultures. Int Dairy J 8(5-6): 535-544

35. Majamaa H, Isolauri E, Saxelin M (1995) Lactic acid bacteria in the treatment of acute rotavirus gastroenteritis. J Pediatr Gastroenterol Nutr 20(3): 333-338

36. Link-Amster H, Rochat F, Saudan KY (1994) Modulation of a specific humoral immune response and changes in intestinal flora mediated through fermented milk intake. FEMS Immunol Med Microbiol 10(1): $55-64$

37. Meydani SN, Ha WK (2000) Immunologic effects of yogurt. Am J Clin Nutr 71(4): 861-872

38. Van't Veer P, Dekker JM, Lamers JWJ (1989) Consumption of fermented milk products and breast cancer: a case-control study in the Netherlands. Cancer Res 49(14): 4020-4023.

39. Sekine K, Watanabe-Sekine E, Ohta J (1994) Induction and activation of tumoricidal cells in vitro and (in vivo by the bacterial cell wall of B infants). Bifidobacteria and Microflora 13: 54-77.

40. Reddy BS, Riverson A (1993) Inhibitory effect of B longum on colon, mammary and liver carcinogenesis induced by 2- amino-3methylimidazo (4, 5-f) quinoline, a food mutagen. Cancer Res 53(17): 3914-3918.

41. Okawa T, NiibeH, Arai T (1993) Effect of LC 9018 combined with radiation therapy on carcinoma of the uterine cervix. Cancer $72(6)$ : 1949-1954.

42. Pradeep K, Kuttapa MA, Prassana Rao (2012) Probiotics and oral health: An update. SADJ 69(1): 20-24.

43. Haukioja A (2010) Probiotics and Oral Health. Eur J Dent 4(3): 348-355.

44. Jain P, Sharma P (2012) Probiotics and their efficacy in improving oral health- A review. J Appl Pharm Sci 2(11): 151-63.

45. Menon AM (2016) Implications of probiotics on oral health: Past-topresent. J Dent Res Rev 3: 36-41.

46. Esha A, Pavan B, Guruprasad CN, Savitha N, Pradeep AR (2011) Probiotics: A novel step towards oral health. Archives of Oral Sciences \& Research 1: 108-115.

47. Naidu KS, Adam JK, Govender P (2012) The use of probiotics and safety concerns: A review. African Journal of Microbiology Research 6(41): 6871-6877.

48. Gasser F (1994) Safety of lactic acid bacteria and their occurrence in human clinical infections. Bulletin de L' Institut Pasteur 92: 45-67. 
49. Rautio M, Jousimies-Somer H, Kauma H (1999) Liver abscess due to a Lactobacillus rhamnosus strain indistinguishable from L. rhamnosus strain GG. Clin Infect Dis 28(5): 1159-1160.
50. Mackay AD, Taylor MB, Kibbler CC, Hamilton-Miller JM (1999) Lactobacillus endocarditis caused by a probiotic organism. Clin Microbiol Infect 5(5): 290-292. (c) (i) Creative Commons Attribution 4.0

For possible submissions Click Here

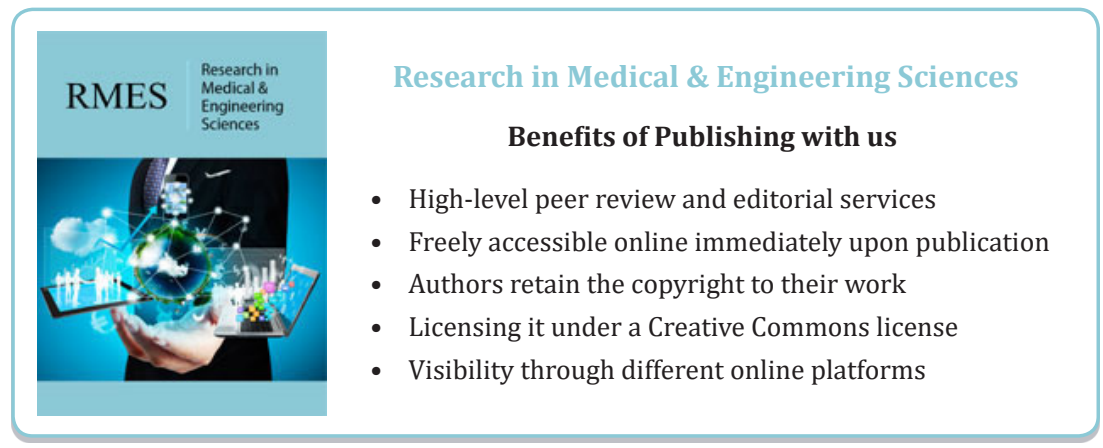

